\title{
A Routing Framework for Delay Tolerant Networks Based on Encounter Angle
}

\author{
Yue Cao, Haitham Cruickshank, Zhili Sun \\ Centre for Communication Systems Research \\ University of Surrey, Guildford, UK \\ \{Y.Cao; H.Cruickshank; Z.Sun\}@ surrey.ac.uk
}

\begin{abstract}
The concept of Delay Tolerant Networks (DTNs) has been utilized for wireless sensor networks, mobile ad hoc networks, interplanetary networks, pocket switched networks and suburb networks for developing region. Because of these application prospects, DTNs have received attention from academic community. Whereas only a few state of the art routing algorithms in DTNs address the problem of aborted messages due to the insufficient encounter duration. In order to reduce these aborted messages, we propose a routing framework which consists of two optional routing functions. Specifically, only one of them is activated according to the encounter angle between pairwise nodes. Besides, the copies of the undelivered message carried by most of the nodes in the network are more likely to be cleared out after successful transfer, which reduces the number of unnecessary transmissions for message delivery. By means of the priority for message transmission and deletion in case of the limited network resource, the proposed algorithm achieves the high delivery ratio with low overhead as well as less number of aborted messages due to the insufficient encounter duration, thus is more energy efficient.
\end{abstract}

\section{INTRODUCTION}

Delay Tolerant Networks (DTNs) [1] are designed to cope with the challenge condition in the restricted networks with sparse network density, intermittent disruption and limited network resource. By means of the store-and-forward mechanism, the message is relayed by the per hop behavior. Routing is a major challenge in DTNs since the characteristic of DTNs prevents traditional routing technique working effectively, which is difficult to maintain the contemporaneously end to end path towards the destination.

In general, the routing protocols must tradeoff the perspective of maximization the message delivery ratio and minimization the resource consumption which are in conflict with each other. On one hand, the ideal approach is to adopt the single copy for successful delivery. However, on the other hand, the effective approach to maximize the message delivery ratio is to enlarge the number of message copies in the networks. Therefore, the feasible approach to reduce the overhead but maintain the high delivery ratio is to intelligently replicate the message.

Bandwidth as an important factor affects the routing performance since it determines the number of messages can be transmitted at each encounter opportunity. In DTNs, message may not be successfully transmitted due to the insufficient bandwidth, which can be interpreted as the limited encounter duration results from the high mobility. Thus the overhead and battery consumption would be increased and critical in the restricted scenario. One feasible approach to overcome this problem is to transmit less number of messages at each encounter opportunity if the encounter angle between pairwise nodes is large since the potential encounter duration is limited. Alternatively, to transmit more messages in case of small encounter angle is acceptable because the smaller encounter angle stands for the longer encounter duration. Another approach is to intelligently delete the message copies after successful transfer since it is unnecessary for the node which does not have high potential approaches the destination to keep on diffusion message. Both of these two approaches are supposed to reduce the number of transmissions for efficient bandwidth usage.

In the following section, we briefly review the state of the art routing algorithms in DTNs, and then in section III we propose our algorithm. Based on the simulation result under realistic scenario in section IV, we draw our conclusion and present our future work in section $\mathrm{V}$.

\section{RELATED WORK}

Except for the algorithms with the assistance of additional nodes such as message ferry or data mule. The early stage of routing algorithms in DTNs are based on the single copy approach [2][3], which forward the message to the candidate node with higher utility metric for the destined node but only one copy of message exists in the network. However its performance degrades in the opportunistic scenario where the message lifetime is quite limited. In general, the multi copy based replication approaches cope with this limitation but result in a high overhead due to the large number of redundancy [4]. Motivated by this drawback, the utility based replication approaches [5] are proposed to alleviate such redundancy.

To our knowledge, the algorithm which predicts the potential encounter duration according to the encounter angle is proposed in [6]. However, its analysis is limited by the assumption that this angle would be maintained constantly from the time when pairwise nodes encounter to the time when communication is disrupted, this might not be realistic under some condition. For example in Fig.1, node $A$ and $B$ encounter with their mobility vectors $V_{A}, V_{B}$ and transmission ranges $R_{A}, R_{B}$. In case 1 , the angle $\theta$ is maintained constantly until the communication between node $A$ and $B$ 
is disrupted. Whereas in case 2 , this angle varies to $\beta$ during the communication time even if the angle at encounter time slot and disruption time slot are equal to $\theta$. From the point of view, the assumption of case 2 is more realstic.

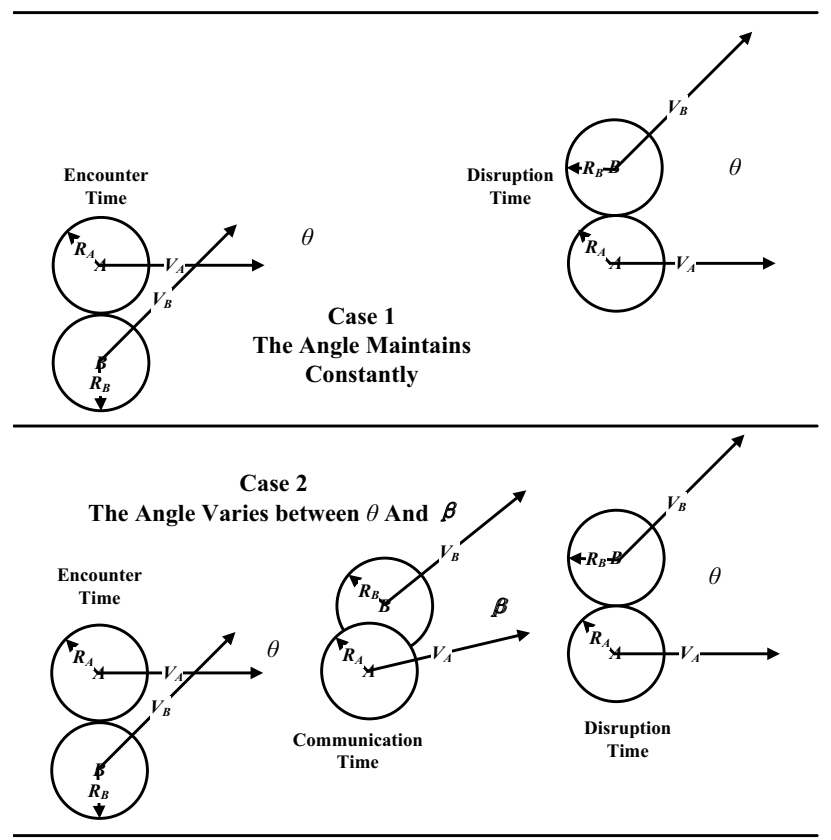

Fig. 1. Two Cases of Encounter

Buffer management plays an important role in DTN routing since the messages suffer from the long delivery delay have to be kept in the limited buffer space until the encounter is available. Therefore, both the priorities used for message transmission and deletion if the buffer space is exhausted significantly affect the routing performance, thus most of the existing routing algorithms have taken into account this issue.

Furthermore, once the message is delivered to the destination, the final receiver generates an acknowledgement and floods this information of which the size is quite small compared with the size of message [7] [8] to reduce the redundancy. The previous approach to reduce the redundancy is passive since it is only activated if the message is delivered. The proactive approach in [9] just focuses on the replication probability but it does not address the issue that under what situation to clear the copy before the final message delivery, which could further reduce the redundancy compared with the passive approach.

\section{Our Proposed Algorithm}

Our proposed algorithm is designed based on the following assumptions:

1: each node can only obtain its location by the GPS system. When pairwise nodes are in communication, by means of the exchanged signaling information they can calculate the encounter angle between each other. To cope with the limitation mentioned in the related work, this angle is calculated for each message transmission, thus it dynamically adjusts to the current situation.

2: the transmission range is quite small compared with the area of the scenario, therefore it is imprecise to obtain the most recent location for the destination by the broadcast information or a centralized location service system.

3: the mobility pattern is unpredictable and movement never stops even if pairwise nodes are in communication.

4: due to the high mobility, the encounter duration is insufficient for the transmission of all the requested messages.

5: the message deletion due to its expiration time is exclude from this paper since we focus on the message loss due to insufficient encounter duration. Therefore the message lifetime is set to be large enough.

The overall function flowchart of our proposed algorithm is illustrated in Fig. 2 and its specific functions are introduced in the following subsections.

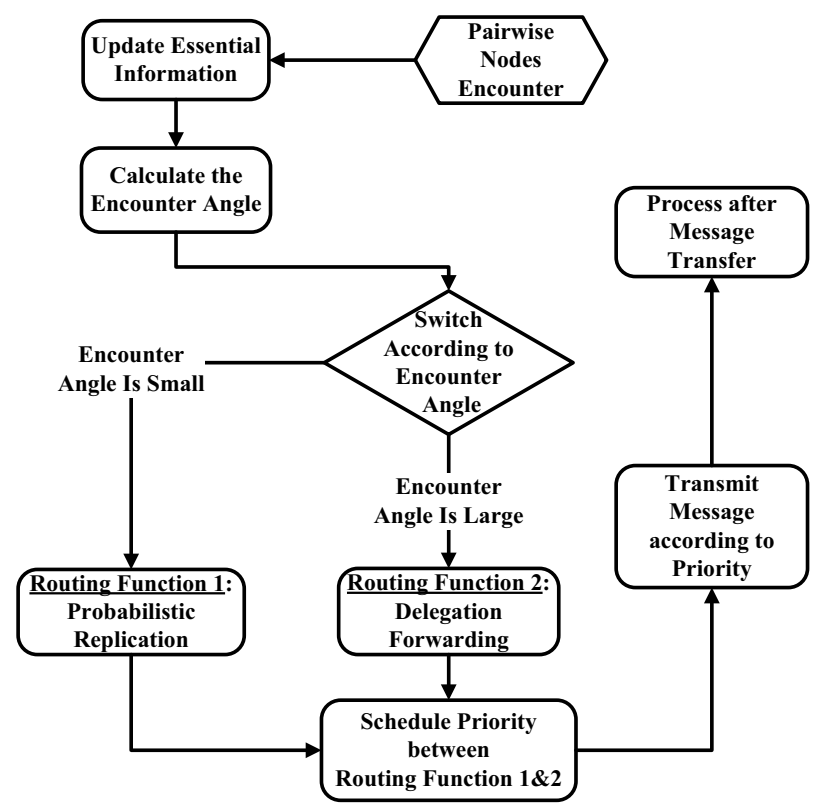

Fig. 2. Function Flowchart

\section{A. Calculation of the Encounter Angle}

Based on our assumptions, each node can obtain its current location by means of GPS system. With an appropriate time window $T$ for the location update, this approach is useful to predict the trajectory of the node. We define the mobility vector to indicate the current trajectory.

Specifically, $\triangle V_{i}$ is the variation of the mobility vector of arbitrary node $i$ within $T$, which consists of the specific variation of 2-D location $\triangle X_{i}$ and $\triangle Y_{i}$. When pairwise nodes are within each other's communication range, the encounter angle $\theta$ between them is calculated by Algorithm 1 .

\section{B. Routing Framework}

Based on the previous calculation of encounter angle, we focus on our routing framework in this subsection. Generally, 




if the encounter angle is quite small, accordingly the potential encounter duration is considered to be longer than the case if the angle is very close to $\pi$. For example, if arbitrary nodes $i$ and $j$ encounter with the angle of 0 or $\pi$. Then the potential encounter duration in case of angle 0 is $\frac{(2 * R)}{|\operatorname{Speed}(A)-\operatorname{Speed}(B)|}$ and the potential encounter duration in case of angle $\pi$ is $\frac{(2 * R)}{|\operatorname{Speed}(A)+\operatorname{Speed}(B)|}$, where $R$ is the minimum transmission range of these pairwise nodes with respective movement speeds Speed $_{A}$ and Speed $_{B}$.

Compared with the analysis in the related work, our proposed approach is effective since the calculation of encounter angle is dynamic for each message transmission. In light of this, we propose to transmit less number of messages if the current encounter angle is large, or to transmit more messages if the angle is small. The key question is to transmit the most appropriate message for the efficient bandwidth utilization. Another benefit from this scheme is to reduce the number of aborted messages due to the high mobility factor, which can reduce the energy consumption and generate less number of messages into the networks with the purpose of congestion avoidance.

Inherently, the utility based replication is an advised scheme if the angle is large because it only replicates the message to the candidate node with higher utility metric compared with the naive flooding scheme such as Epidemic. Recently the delegation forwarding [10] as an algorithm with moderate simplicity and impressive performance has received extensive attention. Its main idea is to cache the utility metric in the message and only replicates the message to the candidate nodes with higher utility than the attached one in the message. For simplicity, we use the encounter count of pairwise encountered nodes $i$ and $j$ as the utility metric $U_{i, j}$ in delegation forwarding and omit the specific algorithm of delegation forwarding in this paper. Specifically, $U_{i, j}$ is the utility for $j$ based on the view of $i$.

Ideally the naive replication scheme achieves better delivery ratio than the utility based replication scheme although it results in the high overhead ratio, thus to adopt the naive replication if the encounter angle is small can compensate the delivery ratio. In particular, although the routing decision is not based on utility metric for the message destination, we still propose a mechanism to optimize this mechanism. In detail, we propose to transmit the message which has not been extensively replicated into the network with higher probability. Hop count is one of the approaches to estimate this state of message. However it can not precisely estimate the replication number of the messages in the network. Firstly, each node would not receive any incoming message that is being carried. Secondly, it might receive the same message again if it dropped this message before, but the hop count of the corresponding message is increased in this case. Motivated by these limitations, we propose to use the carrier count, which is increased by one if the message is received by the node identified by $I D$.

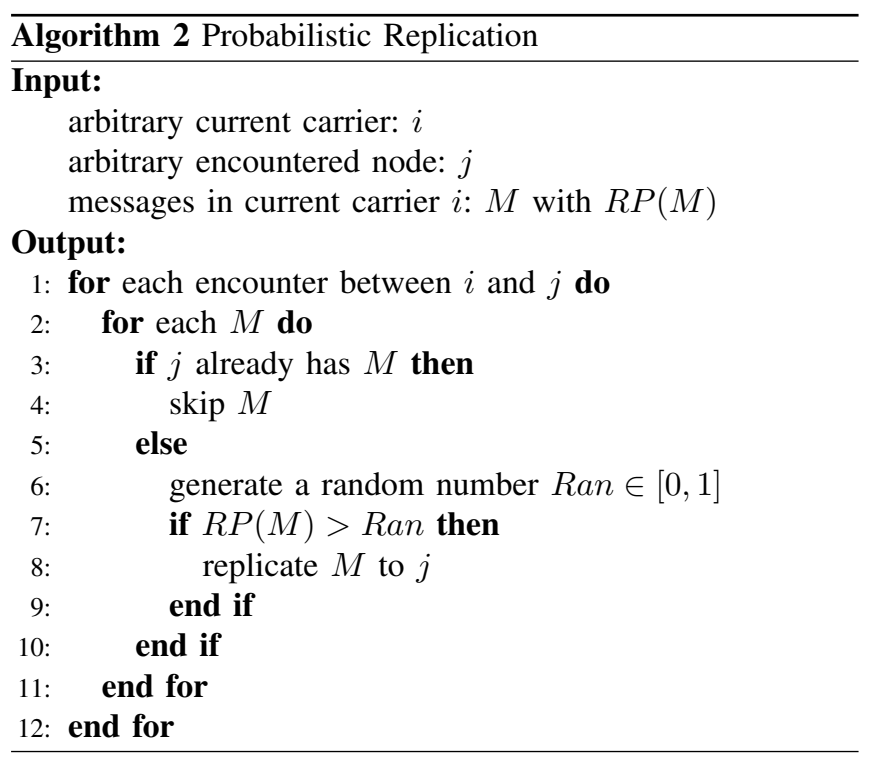

Based on the message (bundle) format of DTNs, we define the carrier count $C(M)$ and utility threshold $T(M)$ cached for each message. $T(M)$ is used in delegation forwarding scheme and the $C(M)$ is used in probabilistic replication.

Regarding the probabilistic replication, we define the replication probability $R P(M)$ by (1), where $N$ is the total number of nodes in the network. The specific algorithm of 
our probabilistic replication is illustrated in Algorithm 2.

$$
R P(M)=1-\frac{C(M)}{N}
$$

When pairwise nodes encounter, both of them exchange the essential information for the delegation forwarding and the probabilistic replication. If pairwise nodes carry the same message $M$, the number of node $I D$ in $C(M)$ of these two carried messages are extended with each other in order to obtain the number of carriers of this message $M$. In addition, regarding the delegation forwarding, the $T(M)$ is updated to the larger value between these two messages.

As we mentioned previously, the encounter angle determines which routing function is activated. An activation probability $A P=\frac{\theta}{\pi}$ is defined for this purpose. The specific routing framework is illustrated in Algorithm 3.

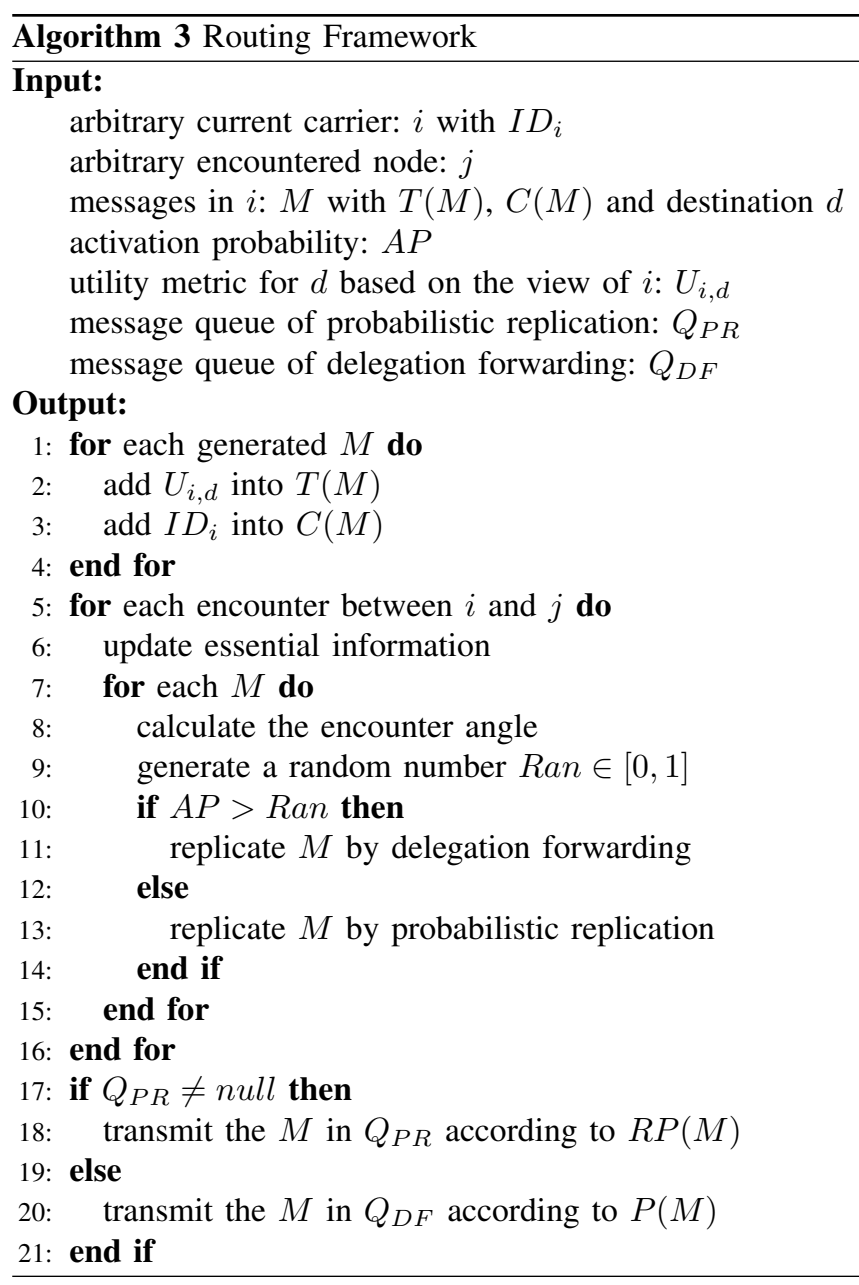

The probabilistic replication can achieve the lower latency since it replicates more messages than delegation forwarding. Based on our framework, it is scheduled prior to delegation forwarding, which focuses on to reduce the latency by scheduling message processed by probabilistic replication with higher priority.
With respect to the priority of message processed by delegation forwarding, its priority $P(M)$ is defined as (2):

$$
P(M)=U_{j, d}-T(M)
$$

where $U_{j, d}$ is the utility value for the destination $d$ of $M$ based on the view of the encountered node $j, T(M)$ is the utility threshold cached in $M$. Therefore, the message which can obtain a larger utility gain is transmitted with higher priority. For the message processed by probabilistic replication, it is transmitted according to its $R P(M)$ since the message with higher $R P(M)$ is supposed it has not been carried by many nodes, thus it is advisable to be allocated with higher priority for transmission.

Inherently, the storage is limited and accordingly each node might not store all of the messages. As a trade-off between the deletion priority for message processed by delegation forwarding and probabilistic replication, the corresponding deletion priority $D P(M)$ is defined as (3):

$$
D P(M)=\frac{T(M)}{(1-R P(M))}
$$

Thus the message with lower utility threshold and lower replication probability is discarded with higher priority.

\section{Process after Message Transfer}

Once the message $M$ is successfully transferred, as it is illustrated in Algorithm 4, both the previous sender and current receiver update $T(M)$. In the meanwhile, the $I D$ of current receiver is added into the $C(M)$. If the message has been carried by most of the nodes in the network, the message in the previous sender has a higher probability to be deleted.

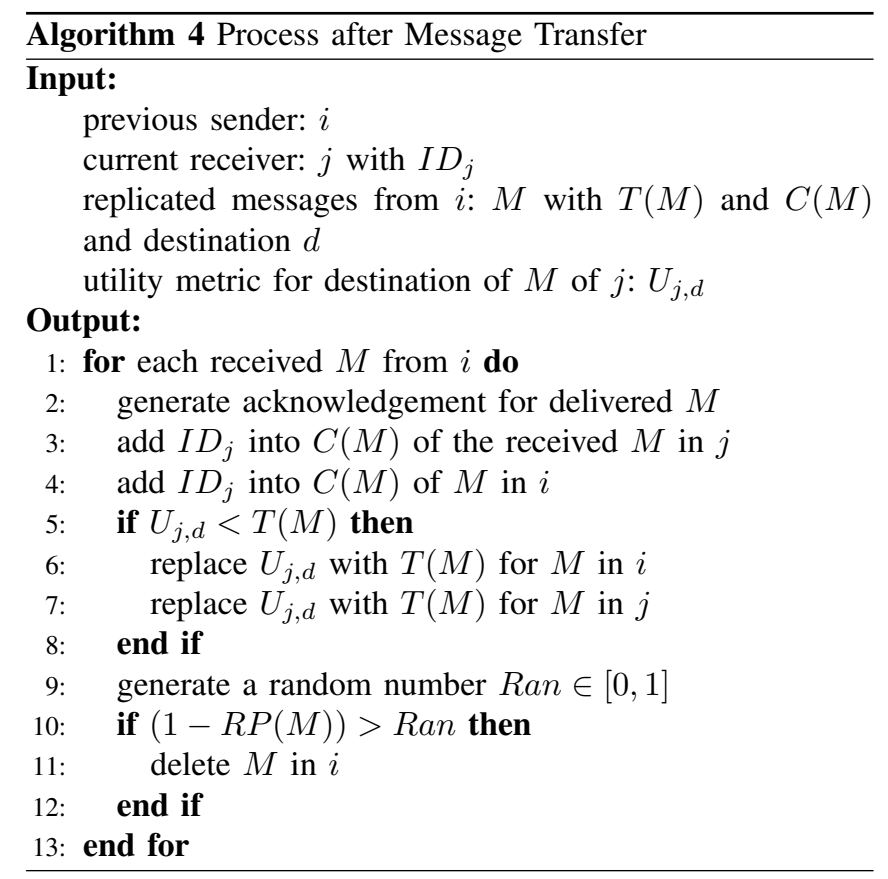

In order to further reduce the redundant transmissions since the intermediate nodes may not obtain the knowledge of 
final messages delivery by their destination. The destination will generate an acknowledgement of which the size can be ignored compared with the size of message when it successfully receives this message and this acknowledgement will be flooded to the entire network. Intermediate nodes receiving this acknowledgement will check their buffer and discard the message which has been successfully delivered.

\section{Simulation Results}

The results are evaluated by Opportunistic Network Environment (ONE). We evaluate the performance of Epidemic, Prophet, MaxProp and also implement an algorithm (we call Adaptive Epidemic) in [9]. The scenario area is $15.3 \mathrm{~km}^{2}$ with 126 mobile nodes configured with different variable speeds. For the purpose of fairness and effectiveness, we evaluate our proposed algorithm with the buffer management to compare with MaxProp. In addition, our proposed algorithm without buffer management is used to compare with the performance of Epidemic, Prophet and Adaptive Epidemic based on FIFO model. In detail, the default parameter configuration for Prophet and Adaptive Epidemic are based on their papers. The battery consumption function is also integrated into all the algorithms and its configuration is based on the NOKIA 6600 smart phone. We run the simulation for 10 times and plot the average value for the results.

TABLE I

Simulation CONFIGURATION

\begin{tabular}{|c|c|}
\hline Simulation Time & $12 \mathrm{Hours}$ \\
\hline Bandwidth & $2 \mathrm{Mb} / \mathrm{s}$ \\
\hline Transmission Range & $10 \mathrm{~m}$ \\
\hline Buffer Size & $10 \mathrm{MB}$ \\
\hline Number of Nodes & 126 \\
\hline Message Size & $500 \mathrm{kB}$ \\
\hline Message Generation Interval & $30 \mathrm{~s}$ \\
\hline Message Life Time & $360 \mathrm{Minutes}$ \\
\hline Initial Energy per Node & $850 \mathrm{~mA} / \mathrm{h}$ \\
\hline Transmission Energy per Node & $51.47 \mathrm{~mA} / \mathrm{h}$ \\
\hline Scanning Energy per Node & $38.61 \mathrm{~mA} / \mathrm{h}$ \\
\hline Time Window & $1 \mathrm{~s}$ \\
\hline Scenario Mobility & Helsinki City Model \\
\hline
\end{tabular}

Regarding the delivery ratio in Fig.3, our proposed algorithm nearly achieves the highest performance which is close to MaxProp even if Maxprop replicates messages without utility consideration. This means our algorithm generates less number of copies than MaxProp but still achieves high delivery ratio. Compared with other algorithms, our algorithm with FIFO still achieves a better performance although is only a little worse than Adaptive Epidemic when the buffer space is large enough.

In Fig.4, it is significant that our proposed algorithm maintains the lowest overhead ratio. One of the contributions is from the routing framework which dynamically determines the corresponding routing algorithm according to the current encounter angle. The buffer management also improves

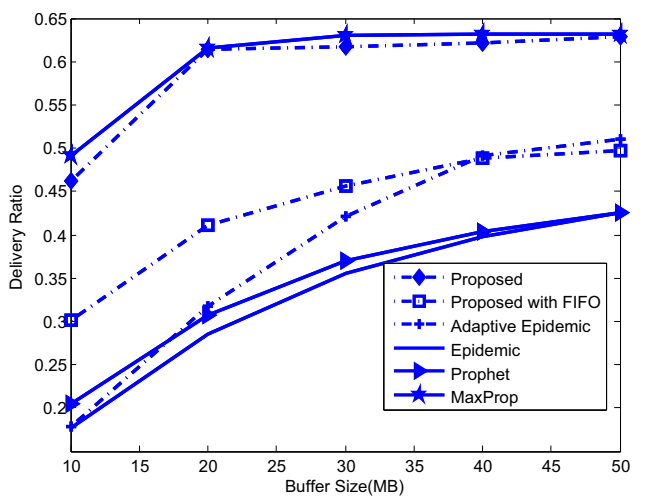

Fig. 3. Delivery Ratio vs Buffer Size

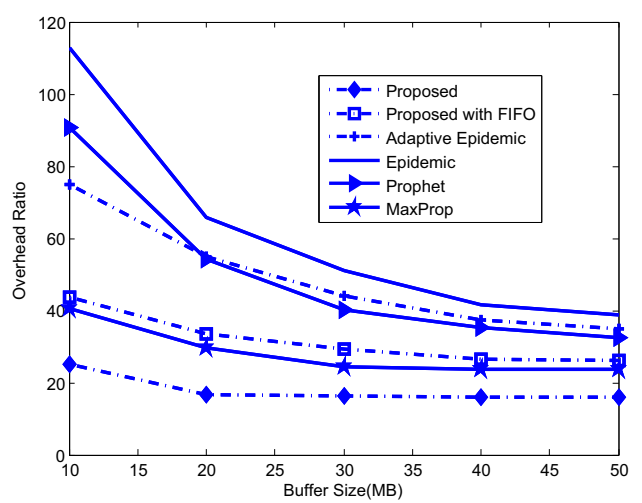

Fig. 4. Overhead Ratio vs Buffer Size

the overhead ratio by determining the priority for message transmission and deletion. Without buffer management, the overhead ratio of our algorithm still outperforms others except MaxProp which is well designed with this function.

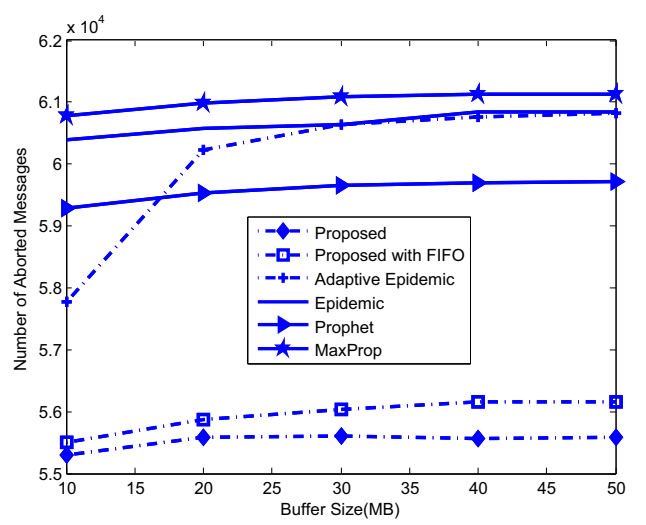

Fig. 5. Number of Aborted Messages vs Buffer Size

Our proposed algorithm dynamically switches the state of routing algorithms between probabilistic replication and delegation forwarding schemes, this mechanism is supposed to limit the number of messages to be transmitted during the 
encounter opportunity. Therefore, even regardless of the buffer management, the proposed algorithm still achieves the least number of aborted messages due to mobility factor in Fig.5. This issue is critical in the high mobility scenario.

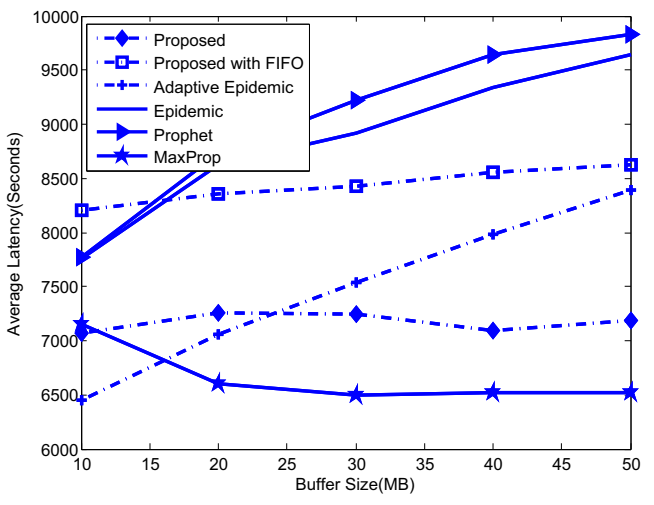

Fig. 6. Average Latency vs Buffer Size

The average latency of our proposed algorithm is a tradeoff between MaxProp and other algorithms in Fig.6. Particularly, with the buffer management, our algorithm maintains the slightly fixed growth of latency whereas the latency of other algorithms without the buffer management are rapidly increased. The lower latency is beneficial though this metric is unimportant in DTNs since its application is regarded to be tolerant to long latency.



Fig. 7. Total Residual Battery vs Buffer Size

We address the residual battery issue in Fig.7 to show the energy consumption. Our proposed algorithm achieves both the higher delivery ratio and less battery consumption. Therefore, it is energy efficient compared with MaxProp which consumes more battery for high delivery ratio.

\section{CONCLUSION}

Taking into account the potential encounter duration based on the consideration of mobility factor is still an open issue even if most of the routing algorithms in DTNs have been proposed in the last few years. In this paper, we address the potential encounter duration issue and propose a routing framework based on the encounter angle. Two optional routing functions are designed based on the decision made by the encounter angle. Our proposed algorithm not only reduces the number of aborted messages due to mobility factor but also achieves the significant performance compared with other state of the art algorithms. In the future, we would extend our work with more intelligent mechanism for the routing framework and further optimize the estimation of potential encounter duration with low complexity.

\section{REFERENCES}

[1] K. Fall and S. Farrell, "Dtn: an architectural retrospective," Selected Areas in Communications, IEEE Journal on, vol. 26, no. 5, pp. 828 $-836,2008$.

[2] S. Jain, K. Fall, and R. Patra, "Routing in a delay tolerant network," vol. 34. New York, NY, USA: ACM, August 2004, pp. 145-158.

[3] E. M. Daly and M. Haahr, "Social network analysis for routing in disconnected delay-tolerant manets," in Proceedings of the 8th ACM international symposium on Mobile ad hoc networking and computing, ser. MobiHoc '07. New York, NY, USA: ACM, 2007, pp. 32-40.

[4] A. Vahdat and D. Becker, "Epidemic routing for partially-connected ad hoc networks," Tech. Rep., 2000.

[5] A. Lindgren, A. Doria, and O. Schelén, "Probabilistic routing in intermittently connected networks," vol. 7. New York, NY, USA: ACM, July 2003, pp. 19-20.

[6] G. Sandulescu and S. Nadjm-Tehrani, "Opportunistic dtn routing with window-aware adaptive replication," in Proceedings of the 4th Asian Conference on Internet Engineering, ser. AINTEC '08. New York, NY, USA: ACM, 2008, pp. 103-112.

[7] A. Balasubramanian, B. Levine, and A. Venkataramani, "Replication routing in dtns: A resource allocation approach," Networking, IEEE/ACM Transactions on, vol. 18, no. 2, pp. 596-609, 2010.

[8] J. Burgess, B. Gallagher, D. Jensen, and B. N. Levine, "Maxprop: Routing for vehicle-based disruption-tolerant networks," in INFOCOM 2006. 25th IEEE International Conference on Computer Communications. Proceedings, 2006, pp. $1-11$.

[9] F. Hou and X. Shen, "An adaptive forwarding scheme for message delivery over delay tolerant networks," in Global Telecommunications Conference, 2009. GLOBECOM 2009. IEEE, 302009.

[10] V. Erramilli, M. Crovella, A. Chaintreau, and C. Diot, "Delegation forwarding," in Proceedings of the 9th ACM international symposium on Mobile ad hoc networking and computing, ser. MobiHoc '08. New York, NY, USA: ACM, 2008, pp. 251-260. 